\title{
Intussusception Secondary to a Meckel's Diverticulum as Leading Point in fourth decade of Life, Case Report
}

\author{
Faisal Albaqami ${ }^{1}$, Feras Alsannaa ${ }^{1}$, Reem Bin Saleem ${ }^{1}$, and Nahla Arab ${ }^{1}$ \\ ${ }^{1}$ Prince Sultan Military Medical City
}

December 22, 2020

\begin{abstract}
37-year-old man presented to the emergency department with a three-hour history of severe, generalized abdominal pain. Computed tomography of abdomen revealed two distal ileoileal intussusceptions with high suspicion of $3 \mathrm{~cm}$ mass within the intussusception around the ileocecal region. Laparoscopic right hemicolectomy with extracorporeal ileocolic anastomosis which showed Meckel
\end{abstract}

\section{Introduction:}

Meckel's diverticulum (MD) is considered the most common gastrointestinal tract (GIT) congenital anomaly. ${ }^{1}$ MD can lead to many complications including bleeding, obstruction, inflammation and perforation. The bowel obstruction may occur through volvulus, intussusception or herniation. ${ }^{2}$ During adult life, intussusception is usually due to malignancy, polyp, stricture or rarely MD. ${ }^{3}$ Here we report a rare case of small bowel intussusception secondary to Meckel's diverticulum in his fourth decade of life.

\section{Case Presentation:}

A 37-year-old middle eastern man presented to the emergency department (ED) with a three-hour history of severe, worsening, generalized abdominal pain and nausea. Past medical history included inactive hepatitis B and morbid obesity. Past family history was unremarkable. Past surgical history included laparoscopic gastric bypass.

On examination, the patient looked unwell, and in pain. His Glasgow coma scale score was 15, and he had a heart rate of 86 beats per minute, blood pressure $129 / 82 \mathrm{~mm} / \mathrm{Hg}$, respiratory rate 20 breaths per minute, and body temperature $36^{\circ} \mathrm{C}$. On examination, his abdomen was lax with generalized tenderness, and his laparoscopic wounds had healed. Body mass index is 27.9 (52 pre surgery).

Initial laboratory investigations revealed a white blood cell count (WBC) of 11,800/uL (4000-11000 uL), and hemoglobin $15 \mathrm{~g} / \mathrm{dL}$ (10-15 g/dL). Other blood investigations were unremarkable. Abdominal X-Ray showed dilated small bowels are noted in the left side of abdomen. Computed tomography $(\mathrm{CT})$ of the abdomen and pelvis with intravenous contrast revealed two distal ileoileal intussusceptions with high suspicion of a $3 \mathrm{~cm}$ mass within the intussusception around the ileocecal region. (Figure 1 ).

He was admitted under the care of the general surgical team, resuscitated, and underwent laparoscopic exploration which confirmed the $\mathrm{CT}$ findings and no ischemic bowel identified. Laparoscopic right hemicolectomy with extracorporeal ileocolic anastomosis was done (Figure 2 ). Upon examining of the specimens Meckel diverticulum was found (Figure 3 ). Specimen was sent for pathology which revealed Meckel's diverticulum lined by gastric mucosa. A solid diet was introduced gradually, and the patient was discharged home on day 8 post operation. He was followed up in the outpatient clinic for almost two years, with uneventful course. 


\section{Discussion:}

When there is one segment of the bowel that invaginates into another portion, this process is named as intussusception. During intussusception, venous outflow will be obstructed due to edema which will lead to ischemia, necrosis and perforation. ${ }^{4}$ The highest incidence of intussusception falls in the first two year of life. It is considered as the most common cause of bowel obstruction during infancy. ${ }^{5}$ However, some studies showed that intussusception can occur at older age and should be considered as differential diagnosis in all age groups (up to $23 \%$ of all intussusceptions). ${ }^{6}$ With aging, the intussusception tends to be due to pathological leading point. Those points including carcinoid tumors, leiomyoma, small bowel lymphoma, vascular malformations, inverted appendiceal stumps or MD. ${ }^{7}$ Furthermore, intussusception's leading point can be part of systematic disease such as Henoch-Schönlein purpura,cystic fibrosis, and hemolytic-uremic syndrome. ${ }^{8}$ MD is considered as the most common gastrointestinal tract (GIT) congenital anomaly. ${ }^{9}$ It is resulted from incomplete obliteration of the vitelline duct which leads to formation of diverticulum. The MD is true diverticulum which containing all layers of small bowel. ${ }^{9}$ Prevalence of MD is estimated up to 2 percent in general population. ${ }^{10}$ Out of this percentage, only approximately 2 to 4 percent of patients develop a complication in their lifetime, specially before two years old. ${ }^{11}$ The rule of two in MD are two inches in length, two feet from the ileocecal valve, two percentage of population and two ectopic mucosa. ${ }^{12}$ The presentation of MD intussusception varies between the age groups, in first two year of life, classic triad of vomiting, abdominal pain, and bloody stools are expected. However, as patient grows the presenting symptoms becoming vaguer. ${ }^{13}$ The complications of MD include bleeding, obstruction, inflammation and perforation. One of the causes of MD bowel obstruction is intussusception. ${ }^{2}$ Since most MDs are asymptomatic and most intussusceptions are of unknown cause, MD causing small bowel intussusception is extremely rare. Despite the rarity of the condition, this case report is demonstrating the importance of considering such diagnosis in this age group. Unlike pediatric intussusception, once the diagnosis of intussusception is made in adult, surgery is the standard of care. Inspection of involved segment should be made, and the resection depends on the viability of the bowel segment. ${ }^{14}$ Back to our case, the diagnosis of MD was not certain, and the involved segment was not healthy therefore resection was done.

\section{Conclusion:}

Although intussusception secondary to MD is rare during adult life and the clinical presentation was not typical, only abdominal pain and isolated nausea, it is important to report such a case and to consider it as differential. Management of MD varies and mainly depends on age of presentation.

\section{Reference:}

1. Jayesh Sagar, Vikas Kumar, D K Shah, Meckel's diverticulum: a literature review. Journal List J R Soc Medv.99(10); 2006 OctPMC1592061.

2. Angela D Levy, Christine M Hobbs, Meckel diverticulum: radiologic features with pathologic Correlation. Mar-Apr 2004;24(2):565-87. doi: 10.1148/rg.242035187.

3. Weilbaecher D, Bolin JA, Hearn D, Ogden W 2nd: Intussusception in adults. Review of 160 cases. Am J Surg 1971;121:531-535.

4. M. D. Stringer, S. M. Pablot, and R. J. Brereton, Paediatric intussusception, British Journal of Surgery, vol. 79, no. 9, pp. 867-876, 1992.

5. Lloyd DA, Kenny SE. The surgical abdomen. In: Pediatric Gastrointestinal Disease: Pathopsychology, Diagnosis, Management, 4th, Walker WA, Goulet O, Kleinman RE, et al (Eds), BC Decker, Ontario 2004. p.604.

6. Cochran, G. L. Higgins III, and T. D. Strout, Intussusception in traditional pediatric, nontraditional pediatric, and adult patients, American Journal of Emergency Medicine, vol. 54, no. 3, p. S31, 2010.

7. J. L. Grosfeld, Intussusception then and now: a historical vignette, Journal of the American College of Surgeons, vol. 201, no. 6, pp. 830-833, 2005.

8. O. Karatepe, M. Tu kenmez, K. Hu nerli et al., Ascaris as a leading point for small-bowel intussusception in an adult: a rare cause of intussusception, American Journal of Emergency Medicine, vol. 26, no. 3, pp. 381e3-381e4, 2008. 
9. Sagar J, Kumar V, Shah DK. Meckel's diverticulum: a systematic review. J R Soc Med 2006; 99:501.

10. T. H. Lee, J. O. Kim, J. J. Kim et al., A case of intussuscepted Meckel's diverticulum, World Journal of Gastroenterology, vol. 15, no. 40, pp. 5109-5111, 2009.

11. M. Yamaguchi, S. Takeuchi, and S. Awazu, Meckel's diver- ticulum. Investigation of 600 patients in Japanese literature, American Journal of Surgery, vol. 136, no. 2, pp. 247-249, 1978.

12. Sagar J, Kumar V, Shah DK (2006) Meckel's diverticulum: a systematic review. J R Soc Med 99: 501-505.

13. R. T. Blakelock and S. W. Beasley, The clinical implications of non-idiopathic intussusception, Pediatric Surgery Interna- tional, vol. 14, no. 3, pp. 163-167, 1998

14. Rashid O, Ku J, Nagahashi M, Yamada A, Takabe K. Inverted Meckel's diverticulum as a cause of occult lower gastrointes- tinal hemorrhage. World J Gastroenterol 2012;18:6155-9.

15. Agha RA, Borrelli MR, Farwana R, Koshy K, Fowler A, Orgill DP, For the SCARE Group. The SCARE 2018 Statement: Updating Consensus Surgical CAse REport (SCARE) Guidelines, International Journal of Surgery 2018;60:132-136.

\section{Figure legends:}

Figure 1; CT of abdomen pelvis revealing evidence of intussusception, with a target sign.

Figure 2; abdomen, ileoceacum, ileo-ileal intussusception

Figure 3; Resected Specimen ( Ileoceacum),Meckel's diverticulum

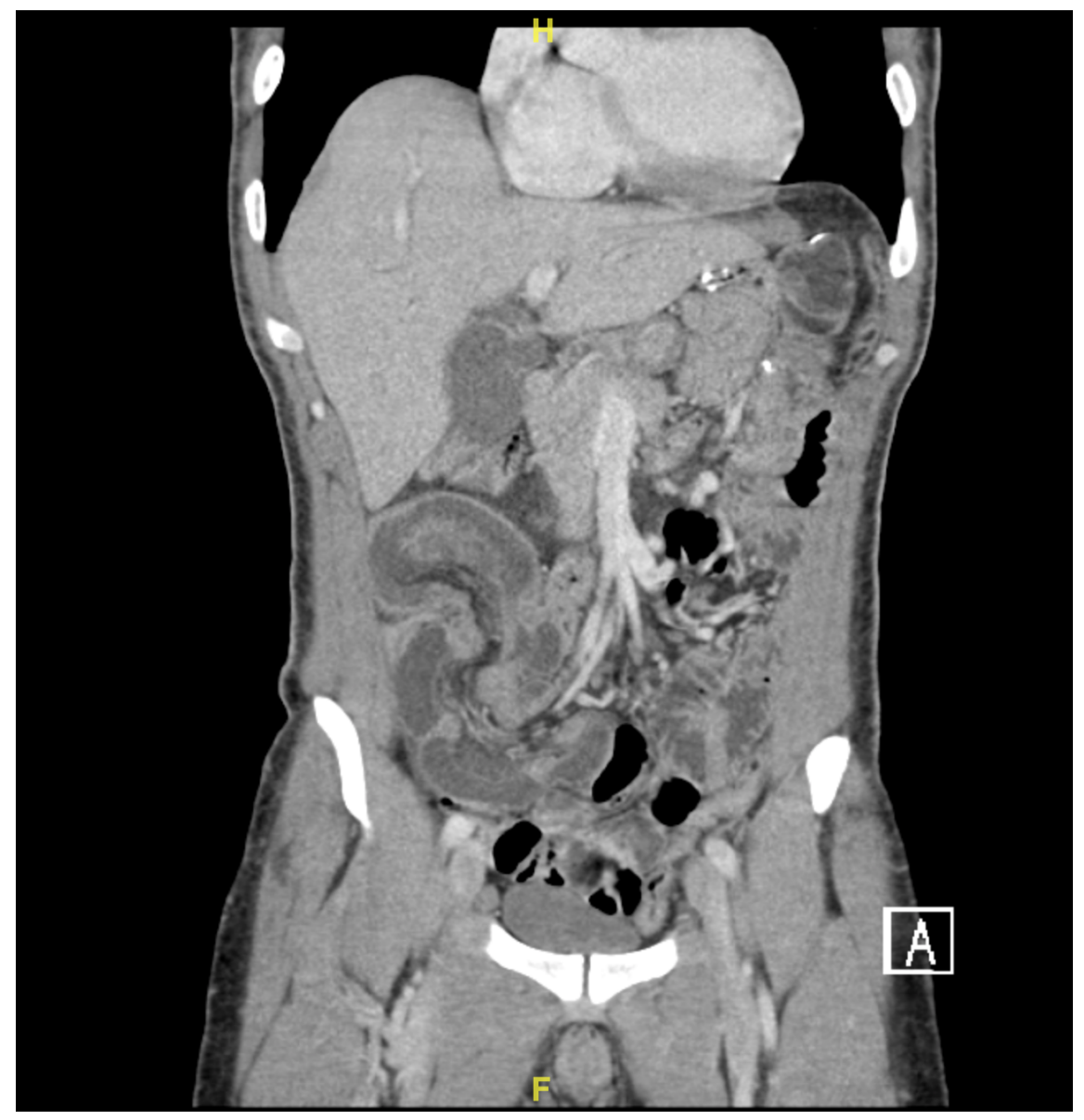



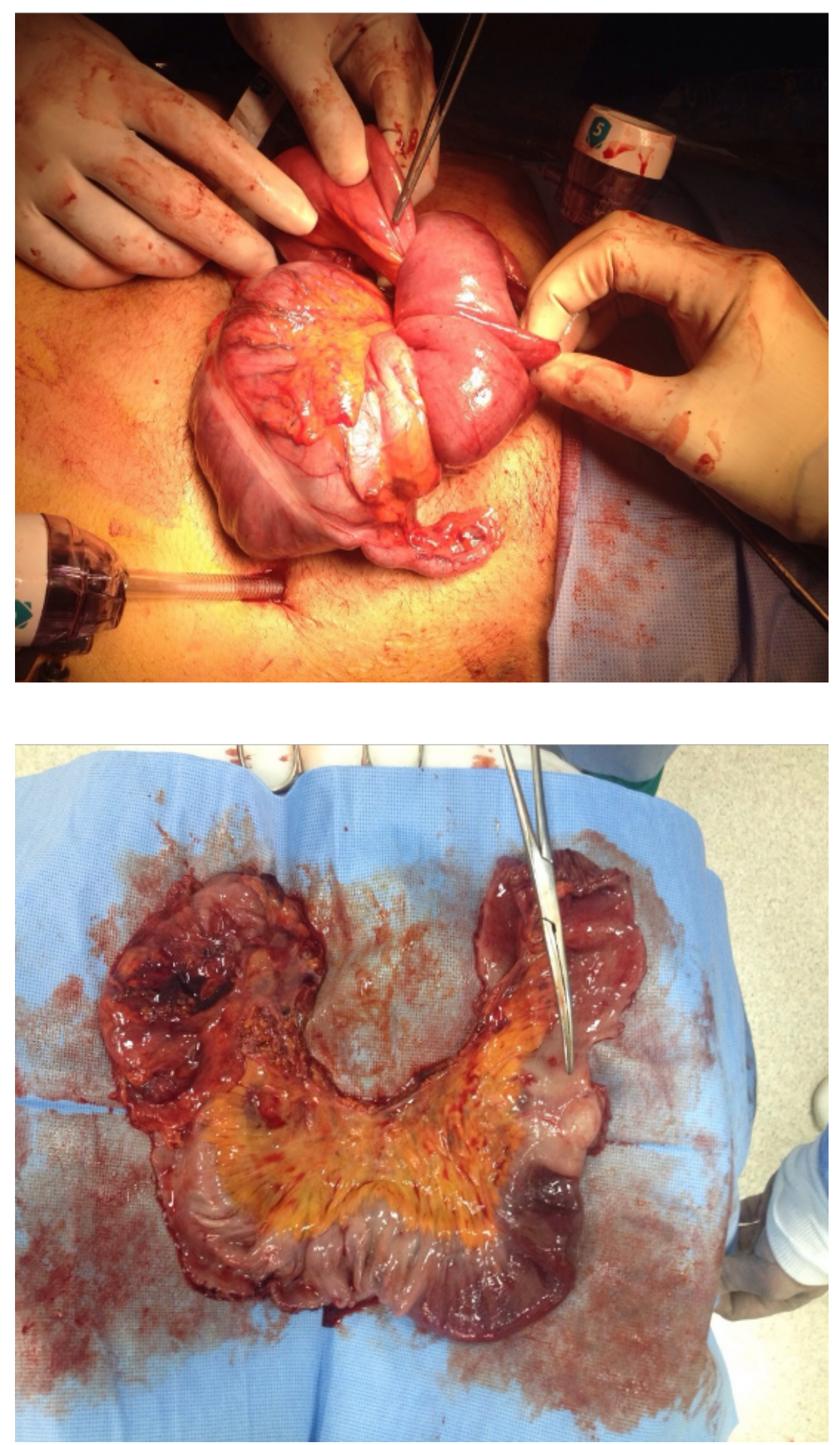